\title{
ÍNDICE AUTORREFERIDO PELA EQUIPE DE CIRURGIA ORTOPÉDICA SOBRE O PROTOCOLO E CHECKLIST DE CIRURGIA SEGURA*
}

\author{
Taysa de Fátima Garcia ${ }^{1}$, Adriana Cristina Oliveira ${ }^{2}$
}

\begin{abstract}
RESUMO: Objetivo: este estudo avaliou o índice autorreferido pela equipe de cirurgia ortopédica quanto ao protocolo de cirurgia segura e aplicação do checklist. Método: estudo descritivo em dois hospitais de Belo Horizonte - Minas Gerais, realizado entre junho e outubro de 2016 com 133 profissionais, médicos (preceptores/ residentes), enfermeiros e técnicos de enfermagem. Não foram incluídos profissionais sob licença médica, férias/ folga durante a pesquisa. Os dados foram analisados descritivamente, por medidas de tendência central e dispersão. Resultados: $83,3 \%$ (25) dos médicos e 95,1\% (98) da equipe de enfermagem afirmaram conhecer o protocolo. Dos cirurgiões, $30 \%$ (9) relataram que o time out é uma prática comum em cirurgias. Esse mesmo percentual afirmou ter vivenciado troca de lateralidade ou retenção de algum material cirúrgico em cavidades operadas. Conclusão: conclui-se que as bases para aplicação do checklist precisam de maior divulgação/monitorização, uma vez que essa lacuna pode comprometer a adoção de ações relevantes impactando na segurança do paciente.

DESCRITORES: Segurança do paciente; Equipe de assistência ao paciente; Conhecimento; Ortopedia.
\end{abstract}

\section{SELF-REPORTED INDEX OF THE ORTHOPEDIC SURGERY TEAM ON THE SURGICAL SAFETY CHECKLIST AND IMPLEMENTATION PROTOCOL}

\begin{abstract}
Objective: The present study assessed the self-reported index of the orthopedic surgery team regarding the safe surgery protocol and application of the checklist. Method: Descriptive study conducted in two hospitals of Belo Horizonte, Minas Gerais, between June and October 2016, with 133 professionals, doctors (mentors/residents), nurses and nursing technicians. Professionals on medical leave, vacation/ holidays were not included in the survey. Data was analyzed with descriptive statistics using measures of central tendency and dispersion. Results: $83.3 \%$ (25) of the physicians and $95.1 \%$ (98) of the nursing workers said they were aware of the protocol. Of the surgeons, $30 \%$ (9) reported that time out is a common practice in surgeries. The same percentage (30) of surgeons said they have experienced laterality errors or retained surgical items inadvertently left in body spaces. Conclusion: it is concluded that the guidelines for application of the surgical safety checklist must to be more widely disseminated/monitored, to ensure that the appropriate actions targeted to patient safety are taken.
\end{abstract}

DESCRIPTORS: Patient safety; Patient care team; Knowledge; Orthopedics.

\section{ÍNDICE AUTORREFERIDO POR EL EQUIPO DE CIRUGÍA ORTOPÉDICA SOBRE EL PROTOCOLO Y CHECKLIST DE CIRUGÍA SEGURA}

RESUMEN: Objetivo: este estudio ha evaluado el índice autorreferido por el equipo de cirugía ortopédica acerca del protocolo de cirugía segura y aplicación del checklist. Método: estudio descriptivo en dos hospitales de Belo Horizonte - Minas Gerais, realizado entre junio y octubre de 2016 con 133 profesionales, médicos (preceptores/residentes), enfermeros y técnicos de enfermería. No fueron incluidos profesionales de licencia médica, vacaciones/día libre durante la investigación. Se analizaron los datos de modo descriptivo, por medidas de tendencia central y dispersión. Resultados: $83,3 \%$ (25) de los médicos y 95,1\% (98) del equipo de enfermería afirmaron conocer el protocolo. De los cirujanos, 30\% (9) relataron que el time out es una práctica común en cirugías. El mismo porcentaje apuntó que hubo cambio de lateralidad o retención de algún material quirúrgico en cavidades operadas. Conclusión: se concluye que las bases para aplicación del checklist necesitan de más divulgación/monitorización, ya que eso puede comprometer la adopción de acciones relevantes impactando la seguridad del paciente.

DESCRIPTORES: Seguridad del paciente; Equipo de asistencia al paciente; Conocimiento; Ortopedia.

\footnotetext{
*Artigo extraído da dissertação intitulada: “Índice autorreferido pela equipe de cirurgia ortopédica sobre as recomendações e diretrizes internacionais e nacionais para a prevenção de infecção do sítio cirúrgico". Universidade Federal de Minas Gerais, 2017.
}

${ }^{1}$ Enfermeira. Mestre em Enfermagem. Enfermeira no Hospital Risoleta Tolentino Neves. Belo Horizonte, MG, Brasil. ${ }^{2}$ Enfermeira. Doutora em Enfermagem. Docente de Enfermagem da Universidade Federal de Minas Gerais.. Belo Horizonte, MG, Brasil. 


\section{INTRODUÇÃO}

A partir da Aliança Mundial para a Segurança do Paciente lançada em 2004, a Organização Mundial da Saúde (OMS) criou em 2008 o programa "Cirurgias Seguras Salvam Vidas" como segundo desafio global, com a perspectiva primordial de melhorar a qualidade da assistência prestada ao paciente cirúrgico ${ }^{(1-3)}$.

Dentre as diretrizes contempladas no programa de cirurgia segura, destaca-se a prevenção de eventos adversos na assistência cirúrgica. Entende-se como evento adverso qualquer lesão ou dano, não intencional, que resulte em incapacidade ou disfunção (temporária ou permanente), e/ou prolongamento do tempo de permanência ou morte, como consequência do cuidado prestado(4).

Estima-se que estes eventos afetem cerca de 3\% a 16\% dos pacientes hospitalizados. Destes, cerca de $3 \%$ em procedimentos cirúrgicos, com taxa de mortalidade global de $0,5 \%$, o que representa que aproximadamente sete milhões de pacientes cirúrgicos estão sujeitos a sofrer alguma complicação a cada ano, e cerca de um milhão pode evoluir com óbito durante ou imediatamente após a cirurgia ${ }^{(1)}$.

No tocante às cirurgias ortopédicas, uma avaliação realizada nos EUA em 2013 identificou que, de 174.167 pacientes submetidos à artroplastia de quadril, 20,6\% deles sofreram no mínimo um evento adverso, destacando-se a infecção em 0,3\%, a ocorrência de fraturas do quadril em 0,3\% e de luxação/ deslocamento do membro em $0,01 \%{ }^{(5)}$.

Nesta perspectiva, uma das recomendações da OMS consiste na utilização de uma lista de verificação cirúrgica - checklist, que tem a finalidade de certificar que aspectos necessários para a realização segura dos procedimentos sejam verificados antes da indução anestésica (sign in), antes da incisão cirúrgica (time out), e antes da saída do paciente da sala de cirurgia (sign out), minimizando assim a possibilidade de ocorrência de eventos adversos ${ }^{(6-8)}$.

O programa cirurgia segura recomenda que a equipe cirúrgica esteja atenta a pequenas ações que, em seu conjunto, são essenciais para a segurança do procedimento cirúrgico. Por outro lado, o esquecimento ou a não observância destas ações podem impactar negativamente, levando à ocorrência de eventos adversos, como cirurgia no paciente errado, identificação incorreta; procedimento cirúrgico errado, em casos que envolvam lateralidade, nível ou estrutura; retenção de objetos na cavidade manipulada, ocorrência de infecção do sítio cirúrgico, dentre outros ${ }^{(3,9)}$.

No entanto, mesmo com os esforços da OMS, tem sido observada na prática a não adesão efetiva dos profissionais ao que é recomendado. Estudos têm demonstrado a ocorrência de eventos adversos associados ao não cumprimento do protocolo de cirurgia segura, bem como a redução destes eventos, quando seu cumprimento é efetivo ${ }^{(3,9,10)}$.

No Brasil, um estudo realizado em dois hospitais do estado do Rio Grande do Norte em 2012, ao revisar a implementação do checklist em 375 cirurgias, observou que em $61 \%$ dos procedimentos o instrumento foi utilizado, no entanto somente $4 \%$ foram adequadamente preenchidos ${ }^{(11)}$.

Muitas vezes, a equipe não adere ao protocolo devido à falta de percepção da importância do checklist na prática cirúrgica. Nesse sentido, o conhecimento da equipe de cirurgia é fundamental para que o protocolo seja implementado de forma adequada e homogênea, sobretudo que seja efetivamente utilizado, o que nem sempre é observado na prática, seja pelo desconhecimento ou pela falta de divulgação quanto à sua importância ${ }^{(11-13)}$.

É importante ressaltar que o cumprimento desse protocolo tem como princípio fundamental a melhoria da comunicação entre os membros da equipe cirúrgica, atentando ao fato de que os eventos adversos são na maioria das vezes evitáveis. Nesse sentido, esse estudo objetivou avaliar o índice autorreferido pela equipe de cirurgia ortopédica quanto ao protocolo de cirurgia segura e aplicação do checklist.

\section{MÉTODO}


Tratou-se de um estudo transversal com delineamento descritivo, realizado no período de junho a outubro de 2016 em Centros Cirúrgicos (CC) de dois hospitais de grande porte situados no município de Belo Horizonte, Minas Gerais, após aprovação pelo Comitê de Ética e Pesquisa, sob parecer de $\mathrm{n}^{\circ}$ 1.686.981, em 12 de agosto de 2016.

A população deste estudo foi constituída pela equipe de cirurgia ortopédica, que neste estudo envolveu cirurgiões ortopedistas (preceptores e residentes), técnicos de enfermagem e enfermeiros do CC dos dois hospitais participantes deste estudo. Foram excluídos do estudo os profissionais que estavam sob licença médica, férias ou folga durante a realização da pesquisa, e aqueles que após a terceira tentativa de abordagem, não foram encontrados ou não responderam ao questionário.

Destaca-se que devido ao baixo número amostral de profissionais médicos e enfermeiros participantes desta pesquisa, optou-se por agrupar preceptores e residentes na categoria "equipe médica", e enfermeiros e técnicos de enfermagem na categoria "equipe de enfermagem", partindo do princípio de que a execução de funções e o conhecimento acerca da segurança do paciente das duas categorias sejam semelhantes.

Os profissionais que aceitaram participar do estudo assinaram o Termo de Consentimento Livre e Esclarecido e posteriormente foi conduzida uma entrevista face a face com questões correspondentes a características sociodemográficas e conhecimento sobre o protocolo de cirurgia segura. O questionário foi fundamentado nas medidas de segurança do paciente recomendadas no protocolo de cirurgia segura da OMS (2009) e previamente submetido à validação de conteúdo, de critério e de constructo.

Foi entregue aos profissionais um caderno composto por questões abertas, permitindo que elaborassem suas respostas, de acordo com seu domínio e conhecimento, sem que fossem induzidos por alternativas propostas. Os dados obtidos foram analisados no programa Stata 14 por análise descritiva e apresentados em tabelas de distribuição de frequência, medidas de tendência central e de dispersão.

\section{RESULTADOS}

Participaram deste estudo 133 profissionais, 71,5\% da amostra total (186) dos dois hospitais, sendo $30(22,5 \%)$ cirurgiões ortopedistas, destes 11 (8,3\%) eram preceptores e 19 (14,3\%) residentes; 10 (7,5\%) enfermeiros e $93(69,9 \%)$ técnicos de enfermagem. Uma parcela de $53(28,4 \%)$ encontraram-se de férias, licenças ou afastamentos e, portanto, não foram incluídos no estudo. As informações referentes às características sociodemográficas estão apresentadas na Tabela 1.

Tabela 1 - Características sociodemográficas dos profissionais participantes do estudo. Belo Horizonte, MG, Brasil, 2016 ( $\mathrm{n}=133)$ (continua)

\begin{tabular}{lcc} 
Variável & $\mathbf{n}$ & $\%$ \\
\hline Sexo & & \\
\hline Feminino & 82 & 61,6 \\
\hline Masculino & 51 & 38,3 \\
\hline Idade* & & \\
\hline$<35$ anos & 62 & 46,6 \\
\hline$>35$ anos & 71 & 53,4 \\
\hline Formação Profissional & & \\
\hline Médico & 30 & 22,5 \\
\hline Enfermeiro & 10 & 7,5 \\
\hline Técnico de Enfermagem & 93 & 69,9 \\
\hline
\end{tabular}




\begin{tabular}{lcc}
\hline Estado Civil & & \\
\hline Solteiro & 50 & 37,6 \\
\hline Casado & 74 & 55,6 \\
\hline Divorciado & 9 & 6,7 \\
\hline Tempo de atuação profissional* $^{*}$ & \\
\hline$<8$ anos & 55 & 41,3 \\
\hline$>8$ anos & 78 & 58,6 \\
\hline Tempo na instituição* & & \\
\hline$<3$ anos & 57 & 42,8 \\
\hline$>3$ anos & 76 & 57,1
\end{tabular}

*Variável categorizada de acordo com a mediana.

Observou-se predomínio de mulheres 82 (61,3\%); idade > 35 anos 62 (53,4\%); 74 (55,6\%) eram casados, $78(58,6 \%)$ possuíam tempo de atuação profissional > 8 anos, e $76(57,1 \%)$ trabalhavam na instituição por um período > 3 anos. O turno de trabalho dominante foi manhã, 49 (36,8\%), seguido do turno vespertino 45 (33,8\%), 10 (7,5\%) à noite e 29 (21,8\%) eram plantonistas. Sobre trabalhar em outra instituição, $103(77,4 \%)$ declararam não trabalhar em outra instituição e 30 (22,5\%) possuíam outro emprego na área, destes, 20 (66,6\%) em mais uma instituição, três (10\%) em duas instituições, cinco $(16,6 \%)$ em três locais e dois $(6,7 \%)$, acima de três.

Dos profissionais entrevistados com formação superior, 12 (40\%) médicos e nove (90\%) enfermeiros declararam ter alguma especialização, destes, dois (16,6\%) dos médicos possuíam mestrado, um (8,3\%) doutorado, e 10 (83,3\%) alguma especialização lato sensu, nenhum enfermeiro se declarou mestre ou doutor.

No tocante ao conhecimento acerca do protocolo de Cirurgia Segura, proposto pela OMS, observou-se que 25 (83,3\%) dos médicos e 98 (95,1\%) da equipe de enfermagem afirmaram conhecer o protocolo. Sobre ter recebido algum treinamento da aplicação do checklist (nos anos de 2015 ou 2016), 17 (56,7\%) e 97 (94,2\%) dos cirurgiões e equipe de enfermagem, respectivamente, mencionaram ter recebido algum treinamento, geralmente aplicado por um enfermeiro 110 (83,3\%). Com relação ao uso do checklist prevenir complicações na cirurgia, $132(99,2 \%)$ do total de entrevistados afirmaram que seu uso previne complicações da cirurgia e 123 (92,5\%) adotam sua aplicação na prática.

Na Tabela 2 estão apresentadas as porcentagens das respostas autorreferidas sobre o protocolo de cirurgia segura por categoria profissional.

Tabela 2 - Índice autorreferido sobre o protocolo de cirurgia segura por categoria profissional. Belo Horizonte, MG, Brasil, 2016 ( $n=133$ ) (continua)

\begin{tabular}{lccc} 
Variável & \multicolumn{3}{c}{ Categoria profissional } \\
\cline { 2 - 4 } & Equipe médica & $\begin{array}{c}\text { Equipe de } \\
\text { enfermagem }\end{array}$ & Total \\
\cline { 2 - 4 } & $\mathbf{n = 3 0 ( \% )}$ & $\mathbf{n = 1 0 3}(\mathbf{\%})$ & $\mathbf{n = 1 3 3}(\mathbf{\%})$ \\
\hline Objetivos do protocolo de cirurgia segura & $\mathbf{1 7}(\mathbf{5 6 , 7 )}$ & $\mathbf{6 7}(\mathbf{6 5 , 0})$ & $84(63,1)$ \\
\hline Tornar procedimento cirúrgico seguro & $3(10)$ & $3(3)$ & $6(4,5)$ \\
\hline Melhorar comunicação da equipe & $4(13,3)$ & $44(42,7)$ & $48(36)$ \\
\hline Reduzir complicações & $0(0)$ & $2(1,9)$ & $2(1,5)$ \\
\hline Reduzir mortalidade & & & \\
\hline Complicações evitáveis com aplicação do checklist & $9(30)$ & $24(23,3)$ & $33(24,8)$ \\
\hline Prevenção da infecção de sítio cirúrgico &
\end{tabular}




\begin{tabular}{lccc}
\hline Operação no paciente errado & $\mathbf{2 0}(\mathbf{6 6 , 8 )}$ & $\mathbf{7 3}(\mathbf{7 0 , 9 )}$ & $\mathbf{9 3}(\mathbf{6 9 , 9 )}$ \\
\hline Operação no local errado & $\mathbf{2 7}(\mathbf{9 0 , 0 )}$ & $\mathbf{7 5}(\mathbf{7 2 , 8})$ & $\mathbf{1 0 2}(\mathbf{7 6 , 7 )}$ \\
\hline Procedimento errado & $12(40)$ & $32(31)$ & $44(33)$ \\
\hline Retenção de materiais & $0(0)$ & $4(3,8)$ & $4(3)$ \\
\hline Falta de comunicação & $5(16,7)$ & $11(10,7)$ & $16(12)$ \\
\hline Erros na administração de medicamentos & $3(10)$ & $28(27,2)$ & $31(23,3)$ \\
\hline Perdas sanguíneas & $0(0)$ & $27(26,2)$ & $27(20,3)$ \\
\hline Revisão de equipamentos & $1(3,3)$ & $12(11,6)$ & $13(9,8)$ \\
\hline Dificuldade para aplicação do checklist & & & \\
\hline Falta de tempo & $6(20)$ & $22(21,4)$ & $28(21)$ \\
\hline Resistência da equipe & $8(26,7)$ & $\mathbf{6 3 ( 6 1 , 2 )}$ & $71(53,3)$ \\
\hline Sem dificuldade & $\mathbf{1 6}(\mathbf{5 3}, \mathbf{3})$ & $18(17,5)$ & $34(25,6)$
\end{tabular}

No tocante à aplicação do checklist de cirurgia segura na prática, quando indagado aos profissionais sobre a realização do processo na instituição, em uma situação em que eles próprios fossem os pacientes, todos os profissionais $(100 \%)$ relataram que gostariam que este fosse realizado. Porém, ao serem questionados se acreditavam que nesta situação o checklist seria realizado, apenas 105 (78,9\%) responderam positivamente.

Ressalta-se que objetivos primordiais do protocolo de cirurgia segura como melhorar a comunicação da equipe obteve baixo índice autorreferido, sendo mencionado apenas por três (10\%) dos cirurgiões e três (3\%) da equipe de enfermagem. Assim como no questionamento acerca das complicações que podem ser evitadas com a aplicação do checklist, apenas quatro $(3,8 \%)$ da equipe de enfermagem relataram que a retenção de materiais na cavidade cirúrgica é uma complicação evitada completamente quando o checklist é utilizado.

Outro achado que chamou atenção foi que 63 (61,2\%) da equipe de enfermagem mencionaram que a resistência da equipe cirúrgica está entre as maiores dificuldades para aplicação efetiva do checklist, enquanto 16 (53,3\%) médicos não apontam dificuldade para o cumprimento do protocolo.

A seguir, o Gráfico 1 apresenta as médias globais para o índice autorreferido pelos profissionais quanto ao protocolo de cirurgia segura.

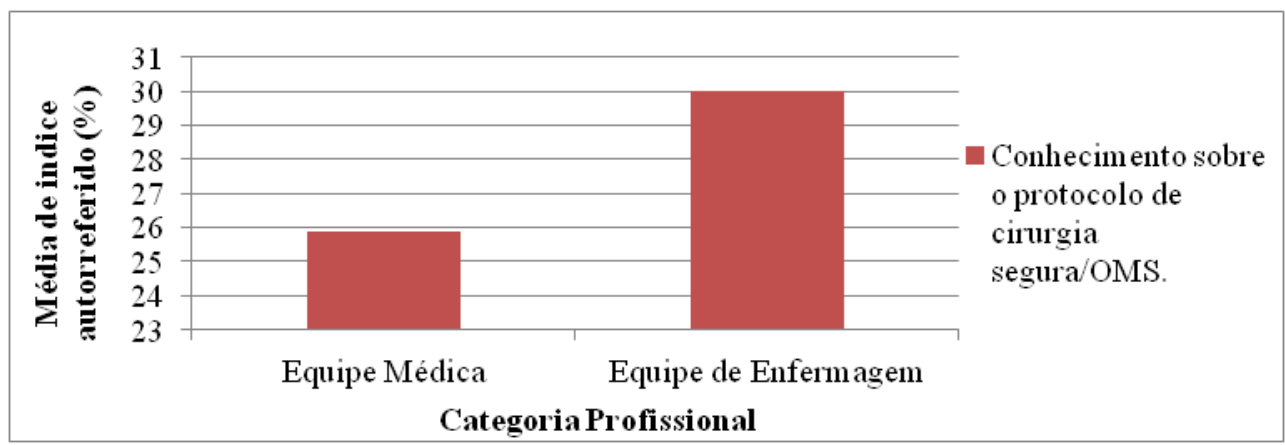

Gráfico 1 - Média global do índice autorreferido pelos profissionais para o protocolo de cirurgia segura, por categoria profissional. Belo Horizonte, MG, Brasil, 2016 ( $n=133$ )

As questões referentes ao time out ou "pausa cirúrgica", contida no checklist de cirurgia segura, foram respondidas exclusivamente pela equipe de cirurgiões ortopedistas, visando indagar sobre seu conhecimento autorreferido e adesão em cirurgias que envolvam lateralidade e colocação de implantes. 
Apenas nove (30\%) médicos relataram que o time out é uma prática comum em cirurgias ortopédicas; $29(96,7 \%)$ afirmaram demarcar o membro a ser operado; nove (30\%) vivenciaram, em algum momento de sua profissão, a troca de lateralidade e retenção de algum material cirúrgico em órgão ou cavidade operadas, como compressas, citado por três $(33,3 \%)$ cirurgiões, gaze por cinco $(55,6 \%)$ ou parafusos mencionado por um $(11,1 \%)$ profissional.

No entanto, 18 (60\%) médicos afirmaram não adotar a contagem de materiais ao final da cirurgia, e, quando realizado, foi atribuído como função de um membro da equipe de enfermagem ou instrumentador, 27 (91,7\%). A análise descritiva dos dados encontra-se na Tabela 3.

Tabela 3 - Índice autorreferido pela equipe médica sobre o time out do checklist de cirurgia segura. Belo Horizonte, MG, Brasil, $2016(n=30)$

\begin{tabular}{lcc} 
Variável & \multicolumn{2}{c}{ Equipe médica } \\
\cline { 2 - 3 } & $\mathbf{( n = 3 0 )}$ & $\mathbf{( \% )}$ \\
\hline Informações confirmadas na pausa cirúrgica & & \\
\hline Identificação do paciente & $\mathbf{1 3}$ & $\mathbf{4 3 , 3}$ \\
\hline Identificação da cirurgia & 9 & 30 \\
\hline Identificação de alergias & 2 & 6,7 \\
\hline Demarcação do local a ser operado & $\mathbf{1 4}$ & $\mathbf{4 6 , 7}$ \\
\hline Disponibilização de exames do paciente & 6 & 20 \\
\hline Apresentação da equipe cirúrgica & 4 & 13,3 \\
\hline Tipo de sinalização adotada para demarcar o sítio cirúrgico & & \\
\hline Marcação de uma "X" no local & $\mathbf{2 3}$ & $\mathbf{7 6 , 7}$ \\
\hline Marcação de uma seta no local & 6 & 20 \\
\hline Escrever no local & 1 & 3,3
\end{tabular}

Com relação ao comportamento da equipe de cirurgiões, quanto à lateralidade, nível ou estrutura a ser operado, quando questionado em que proporção os entrevistados acreditavam que o local da cirurgia era demarcado pelo cirurgião, 13 (43,3\%) dos respondentes mencionaram que o sítio cirúrgico é sinalizado em $100 \%$ das cirurgias; 10 (33,3\%) creem que esta prática acontece entre $70 \%$ a $95 \%$ dos procedimentos e sete (23,3\%) estimam que ocorra apenas entre $10 \%$ a $50 \%$ das cirurgias realizadas na instituição.

\section{- DISCUSSÃO}

Neste estudo, a maioria dos profissionais foram mulheres (61,3\%), especialmente devido ao grande número de profissionais correspondente à equipe de enfermagem, em consonância com outros estudos que reafirmam o predomínio de trabalhadores do sexo feminino em profissões como enfermagem ${ }^{(3,14)}$.

Com relação à faixa etária, a maioria dos participantes possuía idade $>35$ anos (53,4\%) e tempo de atuação profissional de $>8$ anos (58,6\%). Este achado é corroborado com estudos que avaliaram o perfil de trabalhadores de saúde e foram semelhantes a este estudo, com média de idade entre 21 e 60 anos e tempo de atuação de 5 a $10 \operatorname{anos}^{(14-15)}$.

No tocante ao protocolo de cirurgia segura, finalidades como melhorar a comunicação da equipe cirúrgica, apresentou baixo índice autorreferido, sendo mencionado apenas por $10 \%$ dos cirurgiões e $3 \%$ da equipe de enfermagem. A abordagem "Cirurgia Segura salva vidas" visa melhorar a segurança do paciente cirúrgico, reduzir complicações e mortalidade por meio da comunicação efetiva entre a equipe de cirurgia, que representa o seu maior desafio ${ }^{(1,8,16)}$. 
Nesse sentido, o uso do checklist é fundamental para efetivar a comunicação entre os profissionais e na identificação precoce de falhas nos diferentes momentos da assistência ao paciente cirúrgico, apresentando impacto direto na redução de eventos adversos. Estudos têm demonstrado que o uso deste instrumento melhora significativamente a percepção e comunicação da equipe cirúrgica, com impacto direto na assistência e seu comportamento ${ }^{(3)}$.

Sobre demarcar o sítio operatório $76,7 \%$ dos médicos mencionaram o símbolo " $\mathrm{X}$ " para sinalizar o local da incisão, no entanto, 30\% vivenciaram em algum momento de sua profissão a troca de lateralidade. De acordo com o protocolo universal proposto pela Joint Comission, a demarcação do sítio cirúrgico deve evitar qualquer ambiguidade, sendo recomendada a sinalização por meio de uma seta no membro, pois a utilização de um " $X$ " ou de uma cruz no local pode indicar o local a não ser operado, e introduzir confusão no momento da incisão ${ }^{(1,17)}$.

Acerca da realização do time out antes da incisão da pele do paciente, apenas $30 \%$ dos médicos relatou que é uma prática comum em cirurgias ortopédicas. O time out consiste em um dos pontos mais importantes do checklist de cirurgia segura, principalmente por se tratar do momento em que os membros, especialmente o cirurgião, confirmam dados importantes como o paciente a ser operado, a cirurgia a ser realizada e qual o lado a ser operado ${ }^{(17-19)}$.

Estudos têm evidenciado a eficácia da realização do time out na execução multiprofissional do protocolo de cirurgia segura ${ }^{(8,16,19)}$. Ao avaliar a incidência de eventos adversos após a implementação sistemática do time out, um estudo realizado com procedimentos neurocirúrgicos demonstrou que nenhum erro ocorreu em um período de seis meses ${ }^{(18)}$.

Achado similar foi encontrado em um estudo realizado em Los Angeles (EUA), ao incentivar o time out entre 98 membros da equipe cirúrgica, demonstrou que 97,8\% sentiram-se seguros quanto à realização dos procedimentos cirúrgicos após o uso do instrumento ${ }^{(20)}$.

Dentre outros eventos que podem ser evitados com a aplicação do checklist de cirurgia segura, apenas 3,8\% da equipe de enfermagem e nenhum cirurgião relataram que a retenção de materiais na cavidade cirúrgica é uma complicação evitada quando este instrumento é utilizado. Sobre adotar a contagem de instrumentais e materiais ao final da cirurgia, $60 \%$ dos médicos afirmaram não adotar a contagem de instrumentais, e quando esta é realizada comumente é feita pela equipe de enfermagem ou instrumentador $(91,7 \%)$.

Uma comparação entre a realização ou não desta prática, em 59 cirurgias que apresentaram alguma retenção de materiais, identificou-se que a contagem cirúrgica não aconteceu em $6,4 \%$ delas, e em 93,2\% esta prática não ocorreu de maneira correta, resultando em algum evento adverso ${ }^{(21)}$. Em consonância, 30\% dos cirurgiões relataram ter vivenciado, em algum momento de sua profissão, a retenção de algum material cirúrgico em órgão ou cavidade operada, como compressas (33,3\%) gaze $(55,6 \%)$ ou parafusos $(11,1 \%)$.

A retenção de compressas, gases ou instrumentais cirúrgicos na cavidade operatória está comumente relacionada ao porte e caráter da cirurgia (emergências), perda de grande volume de sangue e o envolvimento/participação de muitos membros da equipe cirúrgica no mesmo campo ${ }^{(6-8)}$.

A contagem sistemática de instrumentais e materiais cirúrgicos deve ser realizada pela equipe de cirurgia, com envolvimento multiprofissional, sempre no início e ao final dos procedimentos, com exploração metódica do campo operatório antes do encerramento da cirurgia ${ }^{(1,6-7)}$. Principalmente por se tratar de um processo que permeia toda a cirurgia, desde a montagem da mesa quando os instrumentais são dispostos até o fechamento da incisão.

No entanto, estudos têm demonstrado que a não adesão a esta prática pode resultar em sérios eventos relacionados a retenções de materiais ou instrumentais cirúrgicos na cavidade operatória ${ }^{(22)}$. E pode estar associada ao comportamento de alguns membros da equipe cirúrgica, por acreditar que aspectos relacionados ao material são de responsabilidade apenas da equipe de enfermagem, considerando a falta de tempo de muitos cirurgiões, agenda sobrecarregada e a falsa crença de que tal evento não ocorrerá entre seus pacientes ${ }^{(23-24)}$.

Outro achado que chamou atenção esteve relacionado ao fato de que $61,2 \%$ da equipe de 
enfermagem mencionou que dentre as maiores dificuldades para aplicação efetiva do checklist está a resistência da equipe cirúrgica, enquanto 53,3\% dos médicos não percebem dificuldade para o cumprimento do protocolo.

Outros achados têm demonstrado que a aplicação do checklist é uma prática realizada na maioria das vezes por enfermeiros (78,7\%), seguidos dos anestesistas $(42,5 \%)$, o que reforça a necessidade de maior envolvimento dos cirurgiões(8). Embora o índice autorreferido sobre o protocolo e checklist de cirurgia segura tenha apresentado médias muito aquém do esperado (100\% de acertos) para ambas as categorias, $100 \%$ dos profissionais deste estudo alegaram que gostariam que o protocolo fosse realizado caso se submetessem a alguma cirurgia.

A principal limitação deste estudo foi a dificuldade em acessar a equipe cirúrgica, especialmente a categoria médica, devido à alta demanda de atendimentos cirúrgicos ou ambulatoriais nas instituições, além de imprevistos relacionados ao agendamento cirúrgico, como cancelamentos e/ ou remanejamentos de horários interferindo negativamente no alcance da equipe. Houve ainda as entrevistas que foram iniciadas, mas precisaram ser reprogramadas para outro dia ou horário, e aquelas que foram interrompidas, sem possibilidade de finalização, resultando em perda amostral.

\section{CONCLUSÃO}

No tocante ao índice autorreferido pelos profissionais sobre o protocolo de cirurgia segura, embora a equipe afirme conhecer as recomendações, ao serem questionadas sem apresentação de opções de resposta, as médias ficaram aquém do esperado. Objetivos considerados "padrão-ouro", como a melhoria da comunicação da equipe e a prevenção da retenção de materiais na cavidade cirúrgica, obtiveram índice autorreferido abaixo de 50\%.

Acerca do time out, uma minoria mencionou que esta é uma prática comum entre os cirurgiões ortopedistas e mais da metade dos cirurgiões afirmaram não adotar a contagem de instrumentais ao final da cirurgia. Medidas como a identificação do paciente e do local a ser operado foram reconhecidas como complicações evitáveis com o uso do protocolo apresentando índice autorreferido próximo a $50 \%$.

Nesse sentido, faz-se necessária a discussão multiprofissional de todas as etapas que perpassam a segurança do paciente, sendo imprescindível o investimento em treinamentos envolvendo toda a equipe, reforçando o impacto de suas ações, individuais e coletivas, no cuidado ao paciente cirúrgico.

Ressalta-se que a realização de auditorias de processos, conhecimento e adesão da equipe cirúrgica ao protocolo de cirurgia é de extrema importância e objetiva assegurar a atualização constante dos profissionais, reforçando sua atuação e adesão ao que é recomendado pelas diretrizes para a prevenção da infecção de sítio cirúrgico de maneira integralizada.

\section{REFERÊNCIAS}

1. World Wealth Oganization (WHO). World Alliance for Patient Safety: Safe Surgery Saves Lives. [Internet] Geneva: WHO; 2009 [acesso em 13 abr 2016]. Disponível: http://www.who.int/patientsafety/safesurgery/knowledge_base/ SSSL_Brochure_finalJun08.pdf.

2. Diego LAS, Salman FC, Silva JH, Brandão JC, de Oliveira Filho G, Carneiro AF, et al. Construção de uma ferramenta para medida de percepções sobre o uso do checklist do Programa de Cirurgia Segura da Organização Mundial da Saúde. Rev. Bras. Anestesiol. [Internet] 2016;66(4) [acesso em 9 dez 2016]. Disponível: http://dx.doi. org/10.1016/j.bjane.2014.11.011.

3. Cabral RA, Eggenberger T, Keller K, Gallison BS, Newman D. Use of a Surgical Safety Checklist to Improve Team Communication. AONR J. [Internet] 2016;104(3) [acesso em 8 dez 2016]. Disponível: https://doi.org/10.1016/j. aorn.2016.06.019.

4. Moura MLO, Mendes W. Avaliação de eventos adversos cirúrgicos em hospitais do Rio de Janeiro. Rev. bras. epidemiol. [Internet] 2012;15(3) [acesso em 5 dez 2015]. Disponível: http://dx.doi.org/10.1590/S1415- 
5. Culler SD, Jevsevar DS, Shea KG, Mcguire KJ, Wrigth KK, Simon AW. The incremental hospital cost and lengthof-stay associated with treating adverse events among medicare beneficiaries undergoing THA during fiscal year 2013. J Arthroplasty. [Internet] 2016;31(1) [acesso em 15 out 2016]. Disponível: https://doi.org/10.1016/j. arth.2015.07.037.

6. Motta Filho GR, da Silva LFN, Ferracini AM, Bahr GL. Protocolo de Cirurgia Segura da OMS: O grau de conhecimento dos ortopedistas brasileiros. Rev Bras Ortop. [Internet] 2013;48(6) [acesso em 9 dez 2015]. Disponível: http://dx.doi.org/10.1016/j.rbo.2013.08.002.

7. Russ S, Rout S, Caris J, Mansell J, Davies R, Mayer E, et al. Measuring variation in use of the WHO surgical safety checklist in the operating room: a multicenter prospective cross-sectional study. J Am Coll Surg. [Internet] 2015;220(1) [acesso em 19 jul 2016]. Disponível: https://doi.org/10.1016/j.jamcollsurg.2014.09.021.

8. Candas B, Gursoy A. Patient safety in operating room: Thoughts of surgery team members on implementing the Safe Surgery Checklist (An example from Turkey). Perioperative Care and Operating Room Management. [Internet] 2016;(5) [acesso em 11 jan 2017]. Disponível: http://dx.doi.org/10.1016/j.pcorm.2016.08.001.

9. Beks RB, Claessen FMAP, Oh LS, Ring D, Chen NC. Factors associated with adverse events after distal biceps tendon repair or reconstruction. J Shoulder Elbow Surg. [Internet] 2016;25(8) [acesso em 10 dez 2016]. Disponível: http://dx.doi.org/10.1016/j.jse.2016.02.032.

10. Reed S, Ganyani R, King R, Pandit M. Does a novel method of delivering the safe surgical checklist improve compliance? A closed loop audit. Int J Surg. [Internet] 2016;(32) [acesso em 28 nov 2016]. Disponível: http://dx.doi. org/10.1016/j.ijsu.2016.06.035.

11. de Freitas MR, Antunes AG, Lopes BNA, Fernandes FC, Monte LC, Gama ZAS. Avaliação da adesão ao checklist de cirurgia segura da OMS em cirurgias urológicas e ginecológicas, em dois hospitais de ensino de Natal, Rio Grande do Norte, Brasil. Cad. Saúde Pública. [Internet] 2014;30(1) [acesso em 13 dez 2015]. Disponível: http:// dx.doi.org/10.1590/0102-311X00184612.

12. de Mello JF, Barbosa SFF. Cultura de segurança do paciente em terapia intensiva: recomendações da enfermagem. Texto Contexto Enferm. [Internet] 2013;22(4) [acesso em 30 out 2015]. Disponível: http://dx.doi. org/10.1590/S0104-07072013000400031.

13. Santana HT, de Freitas MR, Ferraz EM, Evangelista MS. WHO Safety Surgical Checklist implementation evaluation in public hospitals in the Brazilian Federal District. J Infect Public Health. [Internet] 2016;9(5) [acesso em 30 dez 2016]. Disponível: https://doi.org/10.1016/j.jiph.2015.12.019.

14. Veríssimo L, Poeira A. Tipologia de perfis socioprofissionais e a identificação profissional numa organização de saúde. Rev Port Saúde Pública. [Internet] 2012;30(2) [acesso em 30 abr 2016]. Disponível: https://doi.org/10.1016/j. rpsp.2012.07.001.

15. Carrillo-García C, Solano-Ruíz MC, Martínez-Roche ME, Gómez-García CI. Influência do gênero e da idade: satisfação no trabalho de profissionais da saúde. Rev. Latino-Am. Enfermagem. [Internet] 2013;21(6) [acesso em 30 dez 2015]. Disponível: http://dx.doi.org/10.1590/0104-1169.3224.2369.

16. Kozusko SD, Elkwood L, Gaynor D, Chagares SA. An innovative approach to the surgical time out: a patientfocused model. AORN J. [Internet] 2016;103(6) [acesso em $12 \mathrm{dez}$ 2016]. Disponível: https://doi.org/10.1016/j. aorn.2016.04.001.

17. The Joint Commission. National Patient Safety Goals Effective January 2017. Office-Based Surgery Accreditation Program. TJC. [Internet] 2017 [acesso em 2 fev 2017]. Disponível: https://www.jointcommission.org/obs_2017_ npsgs/.

18. Oszvald A, Vatter H, Byhahn C, Seifert V, Güresir E. "Team time-out" and surgical safety-experiences in 12,390 neurosurgical patients. Neurosurg Focus. [Internet] 2012;33(5) [acesso em 30 jan 2015]. Disponível: https://doi. org/10.3171/2012.8.FOCUS12261.

19. Molina G, Jiang W, Edmondson L, Gibbons L, Huang LC, Kiang MV, et al. Implementation of the Surgical Safety Checklist in South Carolina Hospitals is associated with improvement in perceived perioperative safety. J Am Coll Surg. [Internet] 2016;222(5) [acesso em 14 dez 2016]. Disponível: https://doi.org/10.1016/j.jamcollsurg.2015.12.052. 
20. McLaughlin N, Winograd D, Chung HR, Van de Wiele B, Martin NA. Impact of the time-out process on safety attitude in a tertiary neurosurgical department. World Neurosurg. [Internet] 2014;82(5) [acesso em 8 mai 2015]. Disponível: https://doi.org/10.1016/j.wneu.2013.07.074.

21. Stawicki SP, Moffatt-Bruce SD, Ahmed HM, Anderson HL, Balija TM, Bernescu I, et al. Retained surgical items: a problem yet to be solved. J Am Coll Surg. [Internet] 2013;216(1) [acesso em 15 fev 2015]. Disponível: http:// dx.doi.org/10.1016/j.jamcollsurg.2012.08.026.

22. Mahran MA, Toeima E, Morris EP. The recurring problem of retained swabs and instruments. Best Pract Res Clin Obstet Gynaecol. [Internet] 2013;27(4) [acesso em 20 set 2014]. Disponível: http://dx.doi.org/10.1016/j. bpobgyn.2013.03.001.

23. Umit UM, Sina M, Ferhat Y, Yasemin P, Meltem K, Ozdemir AA. Surgeon behavior and knowledge on hand scrub and skin antisepsis in the operating room. J Surg Educ. [Internet] 2014;71(2) [acesso em 3 fev 2015]. Disponível: https://doi.org/10.1016/j.jsurg.2013.08.003.

24. Meara JG, Leather AJM, Hagander L, Alkire BC, Alonso N, Ameh EA, et al. Global Surgery 2030: evidence and solutions for achieving health, welfare, and economic development. The Lancet. [Internet] 2015;386(9993) [acesso em 30 mar 2016]. Disponível: http://dx.doi.org/10.1016/S0140-6736(15)60160-X. 